Received March 23, 2015;ACCePted April 8, 2015

Preprint typeset using $\mathrm{LAT}_{\mathrm{E}} \mathrm{X}$ style emulateapj v. 01/23/15

\title{
FIRST SCIENTIFIC VLBI OBSERVATIONS USING NEW ZEALAND 30 METER RADIO TELESCOPE WARK30M
}

\author{
L. Petrov \\ Astrogeo Center, Falls Church, VA 22043, USA
}

T. NATUsch, S. Weston

Institute for Radio Astronomy and Space Research, Auckland University of Technology, Private Bag 92006, Auckland 1142, New Zealand

J. McCallum, S. Ellingsen

University of Tasmania, Private Bag 37, Hobart TAS 7001, Australia

AND

S. Gulyaev

Institute for Radio Astronomy and Space Research, Auckland University of Technology, Private Bag 92006, Auckland 1142, New Zealand Received March 23, 2015;Accepted April 8, 2015

\section{ABSTRACT}

We report the results of a successful 24 hour $6.7 \mathrm{GHz}$ VLBI experiment using the 30 meter radio telescope WARK30M near Warkworth, New Zealand, recently converted from a radio telecommunications antenna, and two radio telescopes located in Australia: Hobart 26-m and Ceduna 30-m. The geocentric position of WARK30M is determined with a $100 \mathrm{~mm}$ uncertainty for the vertical component and $10 \mathrm{~mm}$ for the horizontal components. We report correlated flux densities at $6.7 \mathrm{GHz}$ of $175 \mathrm{radio}$ sources associated with Fermi $\gamma$-ray sources. A parsec scale emission from the radio source 1031-837 is detected, and its association with the $\gamma$-ray object 2FGL J1032.9-8401 is established with a high likelihood ratio. We conclude that the new Pacific area radio telescope WARK30M is ready to operate for scientific projects.

Subject headings: Astronomical Instrumentation

\section{INTRODUCTION}

The New Zealand 30-m radio telescope facility is located near the township of Warkworth, New Zealand, just $5 \mathrm{~km}$ from the Pacific coast. It was built in 1984 as a telecommunication antenna (the Earth Station) to be used by the New Zealand Post Office, later by Telecom New Zealand. In 2010 it was transferred to the Institute for Radio Astronomy and Space Research (IRASR) of Auckland University of Technology for conversion to a radio telescope.

The 30-m antenna has a special wheel-on-track beamwaveguide (BWG) design. This design is often used for deep space network and telecommunications (Imbriale 2005). The feed horn and the receiver are located in the pedestal and do not rotate when the antenna moves along the azimuth and elevation axes. A system of six mirrors directs the beam into the receiver (see Figure 1). The design of the WARK30M antenna should be taken into account for data reduction when dual-polarized data are processed.

Conversion of the 30-m antenna took three years and required a number of upgrades and repairs. Among them was enabling the telescope to track sources with pointing accuracy $1^{\prime}$, connecting the telescope with a 10 Gbps Internet, installation of an uncooled C-band receiver that was donated to IRASR by Jodrell Bank Observatory, installation of a new Digital Base Band Converter (DBBC2) (Tuccari et al. 2010), installation of a Mark5B+ VLBI

Leonid.Petrov@lpetrov.net recorder, connection to the observatories existing Hydrogen maser via a Symmetricom fibre-optic distribution amplifier. Detailed description of the upgrades and the specification of the Warkworth 30-m radio telescope can by found in Woodburn et al. (2015). This work was completed in late 2014, fringe checks were performed and the antenna was ready for its first scientific VLBI experiment.

As a part of commissioning new antennas, the position of the antenna reference point should be determined. A reference point is defined as the point of the projection of the movable elevation axis onto the fixed azimuthal axis. For the analysis of VLBI source imaging experiments made in a phase-referencing mode, position of VLBI stations should be known with accuracy 1020 centimeters to avoid noticeable image smearing ${ }^{1}$ (see e.g. Charlot et al. 2002)). For astrometry applications, angular position accuracies of tens of $\mu$ as are required, making the requirements on the accuracy of station positions much more stringent: $5-10 \mathrm{~mm}$. One way of estimating the position of the antenna reference points is through analysis of a combination of a ground survey of markers attached to the antennas from a local network around the station and GPS observations from the points in the local network (see e.g., Sarti, Sillard \& Vittuari 2004; Sarti, Abbondanza \& Vittuari 2009). At the time of writing this had been planned for the Warkworth $30 \mathrm{~m}$

\footnotetext{
1 See http://www.evlbi.org/user_guide/phrefmp.html for an example of the effect of position errors in quality of image restoration
} 

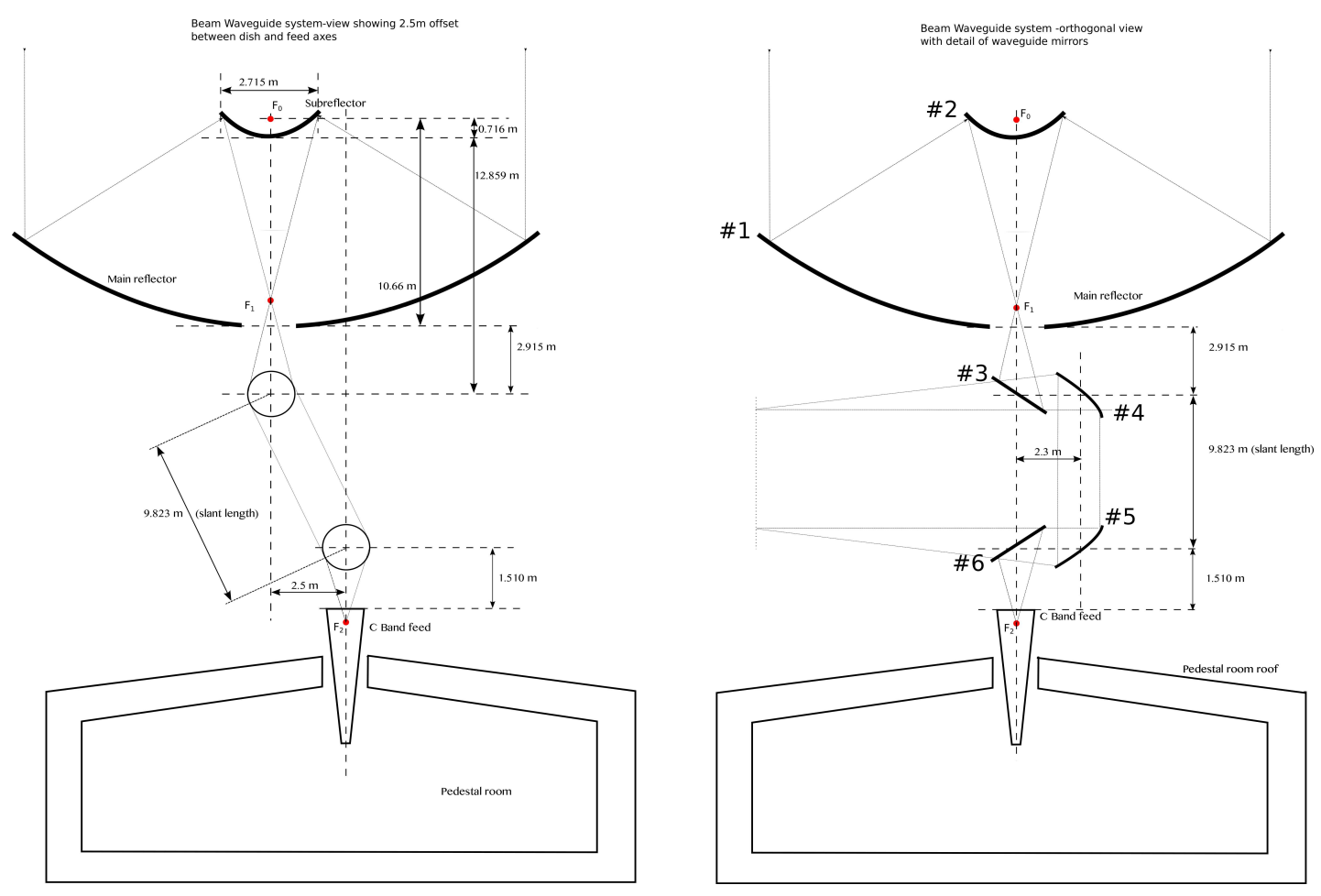

FIG. 1. - WARK30M beam wave-guide diagram in two orthogonal projections (not to scale). Radio waves from the sky are reflected from the main mirror \#1, then reflected from the secondary mirror and guided to the diagonal mirror \#3, which has a circular shape in the plane perpendicular to the elevation axis. The parallel beam formed by this mirror travels $2.3 \mathrm{~m}$ towards the elliptical mirror \#4 that reflects the beam into the waveguide that is tilted at $14.74^{\circ}$ with respect to the vertical. The elliptical mirror \#5 sends the beam to the diagonal mirror \#6, which directs it into the feed horn mounted in the antenna pedestal.

antenna but not carried out. An alternative procedure for estimation of station position is to use the VLBI technique to determine group delays and then derive the reference point position from these group delay measurements. The advantage of the latter approach is that it also provides useful diagnostics of the VLBI equipment and can be combined with astronomical tasks.

Here we report results from the first scientific experiment conducted with WARK30M and two Australian radio telescopes operated by the University of Tasmania in Hobart and Ceduna (McCulloch et al. 2005) on December 11, 2014. The VLBI experiment settings are presented in section 2. The data analysis for both geodetic and astronomical purposes is described in section 3 . Concluding remarks are made in section 4 .

\section{VLBI EXPERIMENT}

Since the WARK30M C-band receiver has a relatively narrow band, approximately $300 \mathrm{MHz}$, our choice of frequency range for VLBI observations was rather limited. We recorded right-circular polarization in a range [6.592, 6.848] GHz. The signal was split into 16 intermediate frequencies (IFs) of $16 \mathrm{MHz}$ each and recorded with 2 bit sampling and an aggregate bit rate 1 Gbps. This setup allows us to determine group delay with formal uncertainty 215 ps when the signal to noise ratio (SNR) is 10 . Two radiotelescopes in Australia participated: CEDUNA and HOBART26 (Figure 2).

We set three goals for the first scientific experiment:

- to determine the position of WARK30M with centimeter level of accuracy;

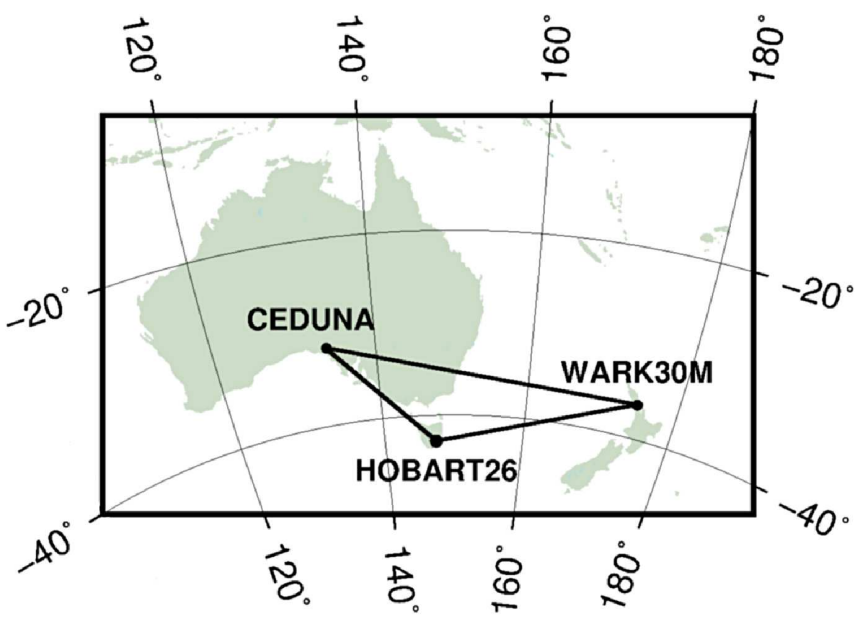

FIG. 2.- VLBI network of this experiment. The map shows locations of the three radio telescopes in New Zealand and Australia and the corresponding baselines.

- to test the effectiveness of the southern VLBI array with WARK30M for monitoring radio flux density of $\gamma$-ray sources;

- to test the usability of the southern VLBI array with WARK30M for association of $\gamma$-ray sources discovered with Fermi by detecting their parsec-scale radio emission.

The pool of 483 targets included all sources from the Radio Fundamental Catalogue ${ }^{2}$ (Petrov and Kovalev,

2 The catalogue is available at http://astrogeo.org/rfc 
2015 , in preparation) that are known to be brighter than $200 \mathrm{mJy}$ at $8 \mathrm{GHz}$ on baselines longer than $5000 \mathrm{~km}$ and are associated with $\gamma$-ray sources. At the moment, $20 \%$ of sources with parsec-scale emission brighter than $200 \mathrm{mJy}$ are known to be $\gamma$-ray loud. Restricting the source list only to these objects did not affect our ability to measure station positions. In total, 244 target sources were scheduled. This includes 7 radio sources from a dedicated Australia Telescope Compact Array (ATCA) 5.5/9.0 GHz survey (Schinzel et al. 2015) never before observed with VLBI. These sources are located within the error ellipse of Fermi objects but marked as "unassociated" in the the 2FGL catalogue (Nolan et al. 2012). As shown by Schinzel et al. (2015), detection of parsecscale radio emission is a powerful method for association of $\gamma$-ray sources. Since the number of compact radio sources is limited, as a rule of thumb, the probability of finding a compact background source with flux density from parsec scales brighter than $10 \mathrm{mJy}$ at $8 \mathrm{GHz}$ within the error ellipse of a $\gamma$-ray object is less than $10 \%$.

Observations were scheduled with software sur_sked ${ }^{3}$ in a sequence that minimizes slewing time and complies with the constraints that (a) the next source is located at a distance not less than $20^{\circ}$ and (b) the minimum temporal separation between consecutive observations of a target source is 3 hours. Target sources were observed for $90 \mathrm{~s}$ each, except for those seven sources in the vicinity of unassociated 2FGL objects: they were observed for $300 \mathrm{~s}$ each. Every hour a sequence of 4 scans of atmosphere calibrators was inserted in the schedule: two scans of sources at elevation angles in a range $\left[15^{\circ}, 30^{\circ}\right]$ and two sources at elevation angles in a range $\left[60^{\circ}, 88^{\circ}\right]$. The purpose of including these scans in the schedule was to improve the robustness of estimation of residual atmospheric path delay in the zenith direction.

\section{DATA ANALYSIS}

The data were transferred electronically to Hobart and correlated with the DiFX software (Deller et al. 2007). Further processing was undertaken with the software package $\mathcal{P} \mathcal{I} M \mathcal{A}^{4}$. We determined the complex bandpass using several strong sources. After applying the bandpass calibration, we performed fringe fitting for each observation, i.e. we determined group delay, phase delay rate, and group delay rate that maximizes the coherent sum of visibilities over time and frequency (Petrov et al. 2011a). The fringe plots for the HOBART26/WARK30M baseline were as expected with no obvious anomalies (See an example in Figure 3). Unfortunately, the receiver frequency response at CEDUNA did not match the response from other telescope receivers. Fringe amplitude for baselines to CEDUNA at frequencies higher than $6.620 \mathrm{GHz}$ dropped by a factor of 10 (Figure 4).

The SNR for baselines to CEDUNA was typically a factor 4 worse than expected, and, therefore, uncertainty in group delays were higher by the same factor. This resulted in the non-detections of $19 \%$ of target sources due to the corresponding sensitivity degradation. Nevertheless, the experiment did not fail, although position accuracy achieved was lower than expected.

\subsection{Geodetic Analysis}

Further processing was made with VLBI analysis software VTD/Post-Solve ${ }^{5}$. After evaluation of group delays from visibility data, the theoretical path delays were computed using the state-of-the art model and small differences between them and the measured group delays were formed. A detailed description of this step can be found in Petrov et al. (2011b).

The a priori slant path delays in the neutral atmosphere in the direction of observed sources were computed through numerical integration of differential equations representing the wave propagation through the heterogeneous media. The four-dimensional field of the refractivity index distribution was computed using the atmospheric pressure, air temperature, and specific humidity taken from the output of the Modern Era Retrospective-Analysis for Research and Applications (MERRA) (Reinecker et al. 2011). The output of the model presents the atmospheric parameters at a grid $0.67^{\circ} \times 0.50^{\circ} \times 72$ pressure levels $\times 6^{h}$. A priori slant path delays in the ionosphere were computed using global ionospheric maps of the total electron content derived from Global Navigation Satellite System observations by the analysis center CODE.

In the initial least square (LSQ) solution, positions of all stations, except HOBART26, were estimated, as well as the coefficients of the expansion for the clock function and the residual atmosphere path delay in zenith direction into the B-spline basis of the 1st degree. During the initial phase of the data analysis, outliers were eliminated and the baseline-dependent corrections to the a priori weights defined to be reciprocal to formal uncertainties of group delays were determined in such a way that the ratio of the weighted sum of squares of residuals to their mathematical expectation was close to unity. The weighted root mean squares (wrms) of postfit residuals was 114 ps on HOBART26/WARK30M baseline and 440 ps on baselines to CEDUNA. The increase of residuals with CEDUNA was caused by the mismatch of the receiver's frequency response.

The final LSQ solution used all VLBI group delays collected from January 1984 through January 2015, in total, 11.4 million values, including 705 group delays from this experiment. Positions of all stations, coordinates of all sources, the Earth orientation parameters, as well as over 1 million nuisance parameters were estimated in a single LSQ run. The differences in velocity of WARK30M and WARK12M was constrained to zero. Station WARK30M has a significant axis offset, the distance between the azimuth fixed axis and the moving elevation axis. We have adjusted that parameter in our VLBI data analysis. Minimal constraints were imposed to require that the net-translation and net-rotation over new position estimates of 44 stations with long history with respect to positions of observations to these stations in the ITRF2000 catalogue (Altamimi, Sillard \& Boucher 2002) be zero. This ensures that the positions of all stations, including WARK30M be consistent with the ITRF2000 catalogue. More details about the parameter estimation technique can be found in Petrov et al. (2009).

The position estimate of WARK30M at epoch 2000.01.01

\footnotetext{
3 See http://astrogeo.org/sur_sked

4 See http://astrogeo.org/web
}

\footnotetext{
5 See http://astrogeo.org/vtd
} 
Fringe phase at HOBART26/WARK30M, source 0605-085

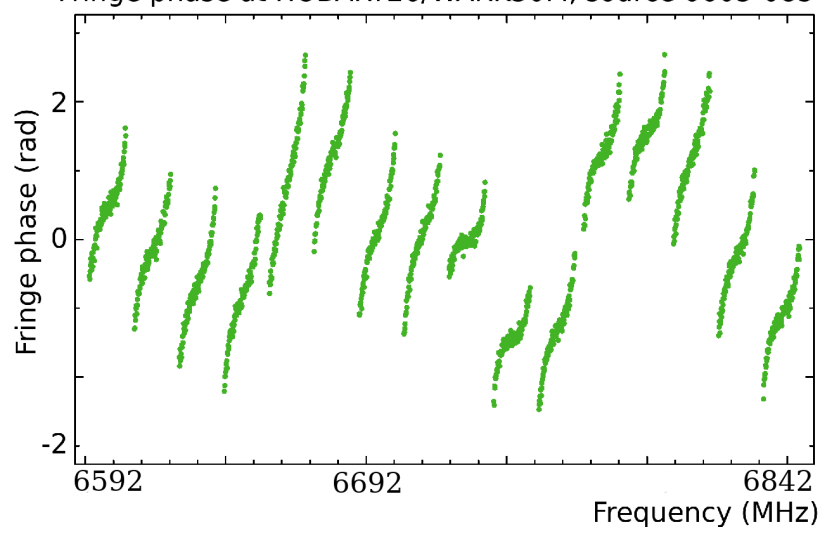

Fringe amplitude at HOBART26/WARK30M, source 0605-085

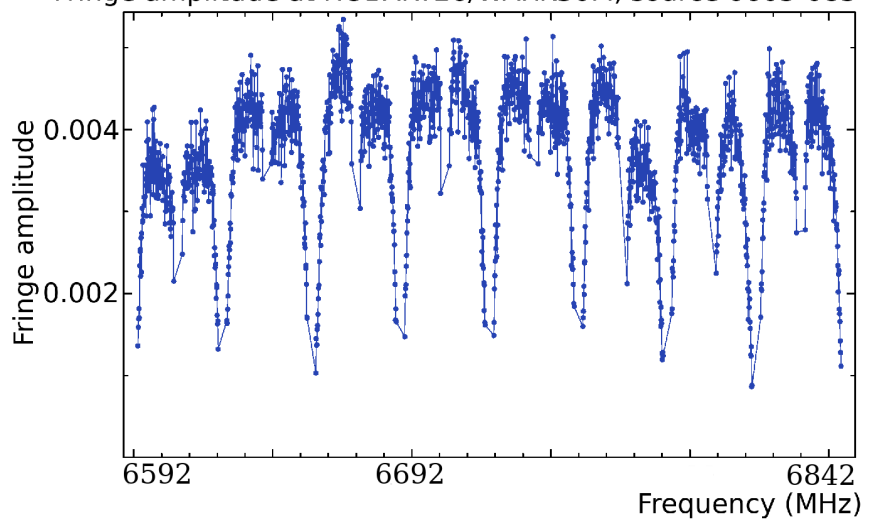

Fig. 3.- Typical fringe phase (Left) and fringe amplitude (Right) at HOBART26/WARK30m baseline before applying the complex bandpass.

Fringe amplutude at CEDUNA/WARK30M, source 0723-008

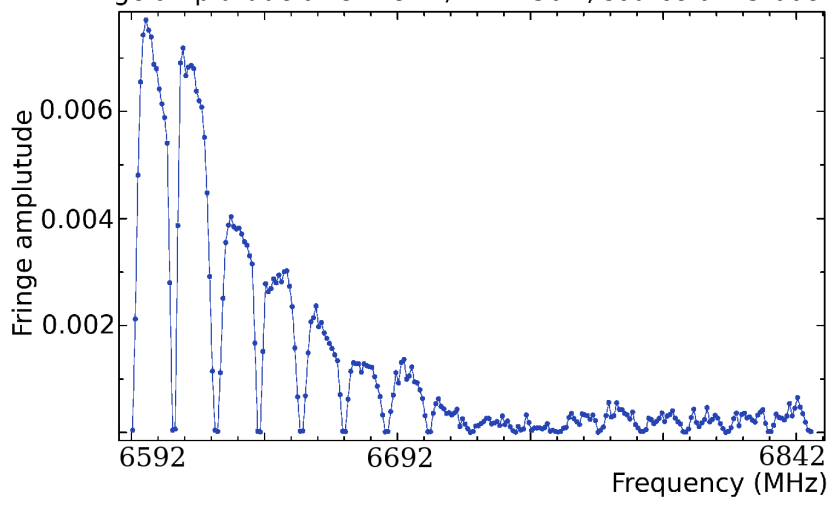

FIG. 4.- Fringe amplitude CEDUNA/WARK30M baseline without applying the complex bandpass. Receiver bands at these two stations do not match.

TABLE 1

COORDINATES AND VELOCITIES OF WARK30M DERIVED FROM ANALYSIS OF THE VLBI EXPERIMENT ON EPOCH 2000.0.

\begin{tabular}{lrl}
\hline $\mathrm{X}$ & $-5115425.60 \pm 0.08$ & $\mathrm{~m}$ \\
$\mathrm{Y}$ & $477880.31 \pm 0.02$ & $\mathrm{~m}$ \\
$\mathrm{Z}$ & $-3767042.81 \pm 0.06$ & $\mathrm{~m}$ \\
Axis offset & $2.61 \pm 0.06$ & $\mathrm{~m}$ \\
$\dot{X}$ & $-19.6 \pm 1.2$ & $\mathrm{~mm} / \mathrm{yr}$ \\
$\dot{Y}$ & $-2.5 \pm 0.5$ & $\mathrm{~mm} / \mathrm{yr}$ \\
$\dot{Z}$ & $34.1 \pm 1.0$ & $\mathrm{~mm} / \mathrm{yr}$ \\
\hline
\end{tabular}

Since velocity of WARK30M was strongly constrained to WARK12M, the reported velocity is based primarily on geodetic observations of WARK12M station.

is provided in Table 1. The errors reported in the table are the formal uncertainties from the LSQ solution computed in accordance with the error propagation law. Projected to the local coordinate system, the uncertainties are 105, 14, and $12 \mathrm{~mm}$ for the Up, East, and North components respectively. High uncertainty in the Up component is due the necessity to estimate antenna axis offset. These two parameters correlate. If we choose not to estimate axis offset, but keep its value to $2500 \mathrm{~mm}$, as specified by the antenna manufacturer, the position uncertainties drop to 38,12 , and $9 \mathrm{~mm}$ respectively.

The largest source of systematic error is the path delay in the ionosphere. In order to evaluate the contri- bution of the residual ionosphere to site position estimates, we re-processed the CONT-14 VLBI campaign with participation of HOBART26 and WARK12M. During 14 days in May 2014 a 17-station array observed a geodetic schedule. We processed fourteen 24-hour experiments independently and estimated baseline lengths between HOBART 26 and WARK12M. We undertook two separate analyses. We used ionosphere-free linear combinations of group delays at 2.3 and $8.4 \mathrm{GHz}$ in the first run. The so-called baseline length repeatability, i.e. the wrms of baseline lengths, was $2.7 \mathrm{~mm}$. In the second run we used group delays at $8.4 \mathrm{GHz}$ and applied the a priori ionosphere contribution computed from the CODE global ionosphere model using the same algorithm as we used for analysis of the experiment with WARK30M. The wrms was $6.2 \mathrm{~mm}$, i.e. the contribution of residual ionosphere to baseline lengths was $5.5 \mathrm{~mm}$ in quadrature. For deriving the contribution of the ionosphere to the vertical component of the WARK30M position estimate, we should scale the repeatability of the $2415 \mathrm{~km}$ long baseline HOBART26/WARK12M by two factors: the geometric factor, the ratio of the Earth's diameter and the baseline length, and the frequency factor: the square of the effective frequency observed in CONT-14 campaign to the square of the effective frequency observed in our experiment. This gives us an estimate of the systematic error in the vertical component of WARK30M position: $50 \mathrm{~mm}$. The systematic error in the horizontal component of position vector is smaller by the geometric factor: $10 \mathrm{~mm}$. This analysis demonstrates that the systematic errors due to the mismodeled ionosphere contribution do not dominate over random errors.

\subsection{Astronomy Analysis}

Among seven target sources in the vicinity of Fermi objects observed in our experiments, one radio source, 1031-837, has been detected. Its coordinates, determined from the global LSQ solution, are $\alpha=10^{h} 30^{m} 15^{s} .286 \pm$ $0^{s} .003, \delta=-84^{\circ} 03^{\prime} 08^{\prime \prime} .652 \pm 0^{\prime \prime} .004$ for the J2000.0 epoch. No $\cos \delta$ factor was applied to the reported uncertainty in right ascension.

At the time of our experiment, concurrent measurements of system temperature were not implemented. The system temperature at different elevations was measured eight days before the experiment, and we found that it fits well to the following regression model: $T_{\text {sys }}=$ $82.6+4.3 / \sin E$, where $E$ is the elevation angle and the 
temperature is in Kelvins. A priori measurements of the system equivalent flux density (SEFD) resulted in the value of $\sim 600 \mathrm{Jy}$.

Since imaging the data of several scans from a threeelement array without concurrent $T_{\text {sys }}$ measurements at one of the antennas is hopeless, we resorted to a simplified amplitude analysis that we have used before for fringe search surveys (Petrov at al. 2012a,b). Using the measured $T_{\text {sys }}$ (a modelled $T_{\text {sys }}$ in the case of WARK30M ) and the a priori gain curves, we computed a priori SEFD for each observation and calibrated fringe amplitudes averaged over time and frequency. In a case of CEDUNA only the fringe amplitude from the first IF $(6.592-6.608 \mathrm{GHz})$ was used. We identified 48 sources with known images from the VLBI Image Database ${ }^{6}$ maintained by the Astrogeo Center. Using these images, we computed the expected fringe amplitude at given baseline projections. We assumed that the image is the same at 8.4 and $6.7 \mathrm{GHz}$. We formed the ratios of the measured fringe amplitude to their expected values and solved for logarithms of antenna-based gain factors. Estimates of the SEFD at $45^{\circ}$ elevation angle are given in Table 2. The anomalously high SEFD at HOBART is due to a failure of the cryogenic equipment that resulted in a system temperature rise to $\sim 200 \mathrm{~K}$ during the experiment.

TABLE 2

Average fitted SEFD at $45^{\circ}$ Elevation angle.

$\begin{array}{lr}\text { CEDUNA } & 890 \mathrm{Jy} \\ \text { HOBART 26 } & 1890 \mathrm{Jy} \\ \text { WARK30M } & 650 \mathrm{Jy}\end{array}$

The error of SEFD estimates is $15 \%$.

Although the flux density of an individual source may change due to variability, the average ratio of the instantaneous flux density at the experiment epoch to its value on the image epoch for the ensemble of 48 sources should be stable. Using a relatively large ensemble for the evaluation of gain factors allows us to estimate their statistical uncertainty: $15 \%$.

TABLE 3

The MEDian CORRELATED FLUX DENSITY AT $6.7 \mathrm{GHz}$ DETERMINED FROM ANALYSIS OF THIS EXPERIMENT.

\begin{tabular}{llllrr} 
IAU Name & IVS Name & $(3)$ & $(4)$ & $(5)$ & $(6)$ \\
\hline J0004-4736 & $0002-478$ & $\mathrm{C}$ & 6 & 0.38 & 0.03 \\
J0012-3954 & $0010-401$ & & 3 & 0.36 & 0.03 \\
J0019+2021 & $0017+200$ & $\mathrm{C}$ & 3 & 1.03 & 0.04 \\
J0038-2459 & $0035-252$ & & 3 & 0.71 & 0.03 \\
J0049-5738 & $0047-579$ & & 3 & 0.68 & 0.03 \\
J0050-0929 & $0048-097$ & $\mathrm{C}$ & 3 & 0.36 & 0.03 \\
J0058-0539 & $0055-059$ & $\mathrm{C}$ & 3 & 0.35 & 0.02 \\
J0058-5659 & $0056-572$ & & 3 & 0.44 & 0.03 \\
\hline
\end{tabular}

(3): Flag "C" denotes the source was used as an amplitude calibrator; (4): The number of observations; (5): Correlated flux density in Jy; (6): uncertainty of correlated flux density in Jy.

Table 3 is published in its entirety in the electronic edition of the journal. A portion is shown here for guidance regarding its form and content.

${ }^{6}$ Available at http://astrogeo.org/vlbi_images

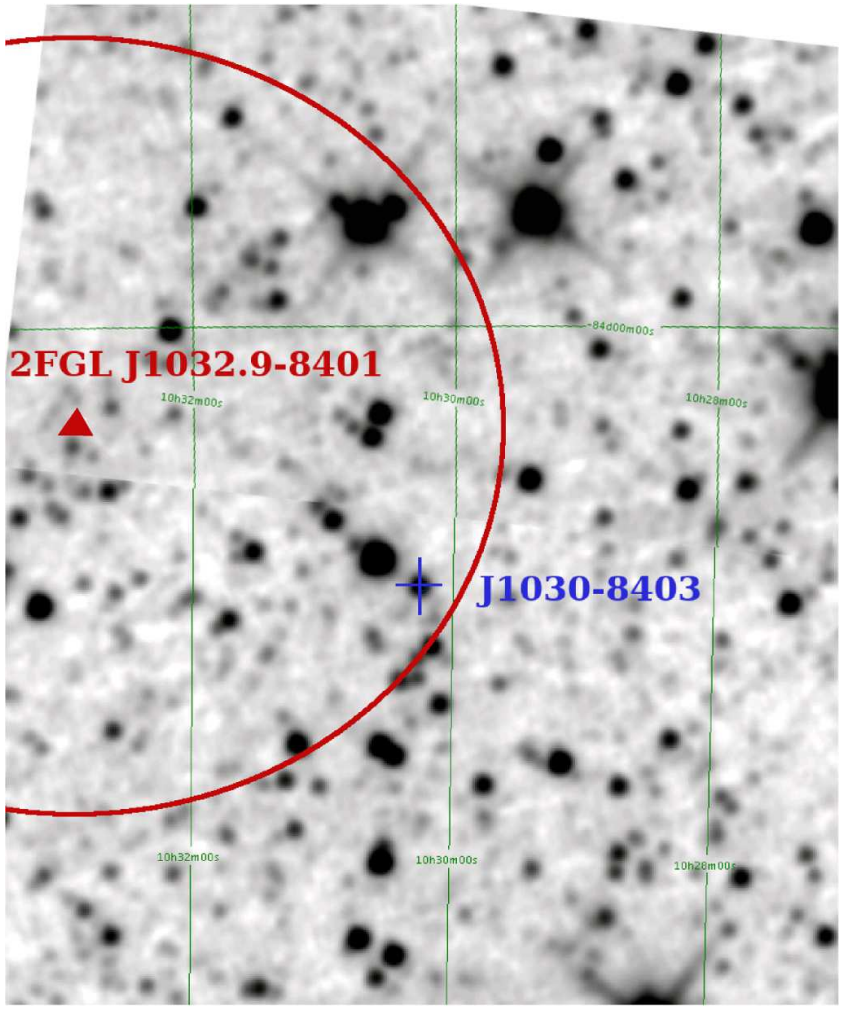

Fig. 5.- Field around 1031-837 at $4.6 \mu \mathrm{m}$ from WISE. The 2$\sigma$ error ellipse around 2FGL J1032.9-8401 position denoted as a triangle is shown with red colour. The blue dot shows 1031-837 position.

Using empirical gain factors, we evaluated the average flux density of each of the 238 sources observed in this experiment. The average flux density of 1031-837 was found to be $46 \pm 7 \mathrm{mJy}$. Since it was detected only in four scans on the HOBART26/WARK30M baseline, no information about its structure can be derived. Nevertheless, using information that we derived from analysis of this experiment, namely, its correlated flux density at projected baseline $50 M \lambda$ and its position, 1.96 Fermi position uncertainty, we were able to compute the likelihood ratio of its association with the $\gamma$-ray object as defined in Schinzel et al. (2015): 22.8. That means that the probability of the $\gamma$-ray source and the radio source being the same object is by a factor of 22.8 greater than the probability of two unrelated objects being incidentally projected close to each other. Thus, we conclude that we have established an association between the radio and $\gamma$-ray sources.

There is a source WISE J103015.28-840308.6 from the Wide-field Infrared Survey Explorer (WISE) catalogue (ALLWISE, November 13, 2013 (Wright et al. 2010; Mainzer et al. 2011)) within 90 mas of 1031-837 position (see Figure 5). Cross-matching the WISE and the Radio Fundamental Catalogue finds 5984 matches. Fitting parameters of the Rayleigh distribution to distances between source positions of matches we found that the estimate of the $1 \sigma$ uncertainty along each coordinate axis is 95 mas. We interpret this estimate as a measure of position accuracy of the ALLWISE catalogue for pointlike sources. Therefore, we conclude that 1031-837 and J103015.28-840308.6 are the same object. It was detected in all 4 bands of WISE instrument with magnitudes of 
$14.10,13.11,10.27$, and 8.16 at $3.4,4.6,12$ and $22 \mu \mathrm{m}$, respectively. Figure 5 demonstrates that the number of IR sources within the Fermi position error ellipse is so high, that their association with the $\gamma$-ray object is impossible without use of additional information.

Analyzing the flux densities of other sources, we found that the typical detection limit for $90 \mathrm{~s}$ integration on the HOBART 26/WARK30M baseline ranged from 40 to $70 \mathrm{mJy}$ with a median value of $50 \mathrm{mJy}$. Fixing the cryogenic problem experienced at HOBART 26 promises to reduce the detection limit to a $20 \mathrm{mJy}$ level.

\section{SUMMARY AND FUTURE WORK}

A new radio astronomy antenna WARK30M has observed successfully its first scientific VLBI experiment. Its position with uncertainty over the vertical coordinate $5 \mathrm{~cm}$ and horizontal coordinates $1 \mathrm{~cm}$ has been determined. Error analysis shows that the systematic errors are a factor of 2 smaller than these uncertainties. The averaged SEFD at $45^{\circ}$ elevation angle was $650 \mathrm{Jy}$.

The first scientific experiment allowed us to establish an association of Fermi detected $\gamma$-ray source 2FGL J1032.9-8401 that was previously considered unassociated. Position of its radio counterpart was determined with the uncertainties of 4.6 mas over both coordinate components and its correlated flux density was found to be $54 \pm 5 \mathrm{mJy}$. We conclude that WARK30M is ready for scientific programs.

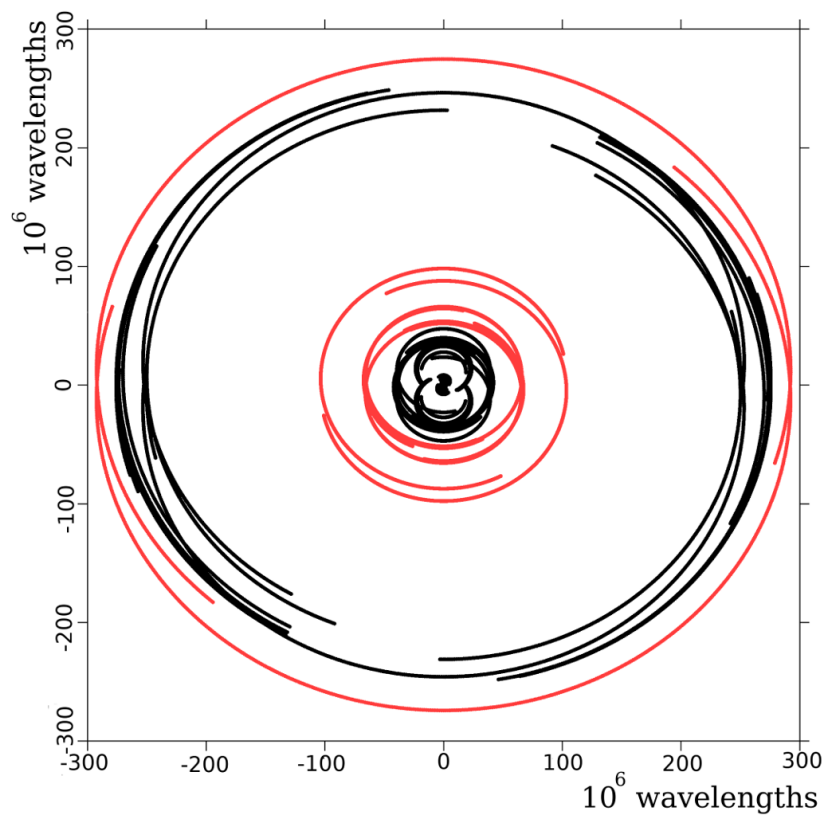

FIG. 6. - The $u v$ coverage of simulated 12 hour long observations of 1934-638 at a 7-station network WARK30M, CEDUNA, PARKES, HOBART26, ATCA, MOpra, and Hartrao. Points at baselines with WARK $30 \mathrm{M}$ are shown with red.

At the moment, there are several VLBI arrays that are actively observing: the Very Long Baseline Array (VLBA), the European VLBI Network (EVN), the VLBI Exploration for Radio Astronomy (VERA), the Korean VLBI Network (KVN), the Chinese VLBI Network (CVN), the Russian Quasar network, and the Long Baseline Array. All these arrays, except the last, are located in the northern hemisphere and cannot observe sources with declinations less than $-40^{\circ}$. WARK30M will be a valuable addition to the network of sensitive southern telescopes (SEFD less than $1000 \mathrm{Jy}$ ): 26m Hartrao, 30m Ceduna, 64m Parkes, 22m Mopra, 6x22m ATCA, and $26 \mathrm{~m}$ Hobart. Figure 6 shows the $u v$-coverage when this seven-station array observes the 1934-638. Tracks of baselines with stations to WARK30M are highlighted in with red. The main focus of VLBI programs with WARK30M is anticipated to be participating in surveys targeting sources in the declination zone $\left[-90^{\circ},-40^{\circ}\right]$ and exploring peculiar objects, mainly in this declination zone inaccessible to the arrays in the northern hemisphere. These programs will be complement to programs that are conducted in the northern hemisphere and will convert them into all-sky surveys. We anticipate that the most valuable contribution of the southern VLBI array with WARK30M participation will be given to those programs that benefit from completeness and all-sky coverage. These programs include the absolute astrometry survey that is used for space navigation, for a connection of VLBI and Gaia coordinate systems, as a list of phase calibrators for phase referencing programs, and for other important applications, such as the VLBI survey of a flux-limited sample of compact radio sources; for a search for associations of $\gamma$-ray sources. We have plans to equip WARK30M with a cooled C-band receiver, an Xband receiver and with the a 2 Gbps recording system in 2015.

Among the 244 observed sources, 175 have been detected in three or more observations. We determined their correlated flux densities with errors 15\% (See Table 3). This shows the potential of a southern hemisphere VLBI array to contribute to programs of flux density monitoring of active galaxy nuclei associated with $\gamma$-ray sources. The TANAMI program of monitoring 84 radio sources associated with $\gamma$-ray objects in the southern hemisphere with the Australian Long Baseline Array (LBA) (Ojha et al. 2010) whose goal is to provide a time series of images. However, TANAMI observes 25-30 sources per 24 hour experiment at a 6-10 station network, i.e. requires more than one order of magnitude more resources. A program of coarse flux density monitoring of a much wider list will be complimentary to TANAMI. By March 2015, there are 270 known $\gamma$-ray sources with declinations $<-30^{\circ}$ associated with the radio sources that exhibit emission from milliarsec regions detected with VLBI. All these sources can be observed in a 27-30 hour observing session. A program of monthly monitoring of these sources has the potential to check their variability and derive light curves.

Both VLBI and single-dish spectral observations in Cband can potentially include spectral line observations of $\mathrm{OH}$ maser lines at 6.03 and $6.035 \mathrm{GHz}$ and methanol masers at $6.7 \mathrm{GHz}$, which are important for the identification and study of star-forming regions. For example, 6.7 GHz methanol masers are exclusively associated with the early stages of high-mass star formation and VLBI observations can be used to obtain accurate distance estimates through trigonometric parallax. Single dish observations with the $30 \mathrm{~m}$ radio telescope in New Zealand can be used to monitoring of the variability of methanol maser sources found by the Parkes multi-beam survey (Woodburn et al. 2015). 


\section{ACKNOWLEDGMENTS}

This research has made use of the data products from the Wide-field Infrared Survey Explorer and the NASA/ IPAC Infrared Science Archive, which is operated by the
Jet Propulsion Laboratory, California Institute of Technology, under contract with the National Aeronautics and Space Administration. We thank Dimitri Duev for careful checking numeric tables.

\section{REFERENCES}

Alexander, F. E. S., 1946, Radio \& Electronics, 1(1), 16-17, 20

Altamimi, Z., Sillard, P., Boucher, C., 2002, J. Geophys. Res., 107, 2214

Bolton, J. G., Stanley, G. J. \& Slee, O. B., 1949, Nature, 164(4159), 101

Charlot, P., et al., 2002, in the 6th EVLBI Network Symposium, 9

Deller, A. T., Tingay, S. J., Bailes, M. \& West, C., 2007, PASP, 119,318

Gulyaev, S., Natusch, T., Addis, B., Tingay, S., Deller, A., 2005, Southern Stars, 44(4), 12

Gulyaev, S. \& Natusch, T., 2009, In International VLBI Service for Geodesy and Astrometry 2008 Annual Report, NASA/TP-2009-214183, eds. Behrend, D. \& Baver, K. D., 68 ftp://ivscc.gsfc.nasa.gov/pub/annual-report/ 2009/pdf/nswark.pdf

Imbriale W. A. 2005, Large Antennas of the Deep Space Network. John Wiley \& Sons, Inc., Hoboken, New Jersey, 313

Mainzer, A., Bauer, J., Grav, T., et al. 2011, ApJ, 731, 53

McCulloch, P. M.; Ellingsen, S. P.; Jauncey, D. L.; Carter, S. J. B.; Cimo, G., Lovell, J. E. J., Dodson, R. G., 2005, AJ, 129(4), 2034

Nolan P. L. et al., 2012, ApJS, 199, 31

Ojha, R. et al. 2012, TANAMI: tracking active galactic nuclei with austral milliarcsecond interferometry. I. First-epoch 8.4 $\mathrm{GHz}$ images, A\&A, 519A, 45O

Petrov, L., Gordon, D., Gipson, J., MacMillan, D., Ma, C., Fomalont, E., Walker, R. C., Carabajal, C., 2009, J Geodesy, 83(9), 859

Petrov L., Kovalev Y. Y., Fomalont E., Gordon D. 2011a, AJ, 142, 35

Petrov, L, Phillips, C., Tzioumis, T., Stansby, B., Reynolds C., Bignall, H. E., Gulyaev S., Natusch T., Palmer N., Collett D., Reynolds J. E., Amy S. W., Wayth R., Tingay S.J., PASA, 2011b, 28(2), 107-116
Petrov, L., Honma, M., Shibata, S. M. 2012a, AJ, 143, 35 Petrov L., Lee S.-S., Kim J., Jung T., Oh J., Sohn B. W., Byun D.-Y., Chung M.-H., Je D.-H., Wi S.-O., Song M.-G., Kang J., Han S.-T., Lee J.-W., Kim B. G., Chung H., Kim H.-G., 2012b, AJ, 144, 150

Rienecker, M. M., Suarez M. J., Gelaro R., Todling R., Bacmeister J., Liu E., Bosilovich M. G., Schubert S. D., Takacs L., Kim G.-K., Bloom S., Chen J., Collins D., Conaty A., da Silva A., 2011, J. Climate, 24, 3624-3648

Sarti, P., Sillard, P., \& Vittuari, L. 2004, J Geodesy, 78(3), 210

Sarti, P., Abbondanza, C. \& Vittuari L., 2009, J Geodesy 83(11), 1115

Schinzel F., Petrov L., Taylor G., Mahony E., Edwards P., Kovalev Y. 2015, ApJS, in press, http://arxiv.org/abs/1408.6217

Tingay, S. J. et al., 2006, ATNF Newsletter, 58, 8-10

Tuccari, G., Alef, W., Bertarini, A., Buttaccio, S., Comoretto, G., Graham, D., Neidhardt, A., Platania, P. R., Russo, A., Roy, A., Wunderlich, M., Zeith, R., Xiang, Y., DBBC VLBI2010, 2010, In Proceedigs of the IFS General Assembly, 28

Weston, S., Natusch, T., Gulyaev, S., 2010, in Proceedings of the 17th Electronics New Zealand Conference, http://arxiv.org/abs/1011.0227

Woodburn, L. et al., 2015, PASA, in press, http://arxiv.org/abs/1407.3346

Wright, E. L., Eisenhardt, P. R. M., Mainzer, A. K., et al., 2010, AJ, 140, 1868 